\title{
Seed Potato Growth and Yield as Affected by Mother Plant Exposure to Herbicides
}

\author{
Jed B. Colquhoun, Daniel J. Heider, and Richard A. Rittmeyer*
}

In a repeated multi-year study, mother potato plants were exposed to herbicides at rates that simulated off-target application, such as through tank contamination. Following exposure of mother plants to herbicides, seed from mother plants was planted in the following growing season and crop growth, yield and tuber quality were quantified. Visual injury from herbicides was observed both in the mother plant and daughter tuber growing seasons and occasional impacts on tuber yield were noted. However, an inconsistent relationship was observed for herbicide related injury and tuber yield reductions of mother potato plants with daughter tuber growth and yield. The lack of consistency in the relationship between visual potato injury in the mother plant production and adverse daughter tuber growth and yield in the following year challenges traditional crop scouting as a tool to predict off-target herbicide risk near seed potato production.

Nomenclature: 2,4-D; aminopyralid; cloransulam-methyl; dicamba; flumiclorac; fluthiacet; glyphosate; mesotrione; metribuzin; metsulfuron-methyl; $S$-metolachlor; tembotrione; thifensulfuronmethyl; topramezone; tribenuron-methyl; potato, Solanum tuberosum L.

Key words: herbicide sprayer tank contamination, daughter tuber.

\begin{abstract}
En un estudio repetido varios años, plantas madres de papa fueron expuestas a herbicidas a dosis que simularon aplicaciones accidentales, tales como las que se dan por contaminación en el tanque. Después de la exposición de las plantas madre a los herbicidas, semilla de estas plantas madre fue plantada en la siguiente temporada de crecimiento y se cuantificó el crecimiento del cultivo, el rendimiento y la calidad de los tubérculos. El daño visual causado por los herbicidas fue observado tanto en las temporadas de crecimiento de la planta madre como el de las plantas hijas y ocasionalmente se notó un impacto en el rendimiento de tubérculos. Sin embargo, se observó una relación inconsistente entre el daño y reducciones en el rendimiento de tubérculos en las plantas de papa madre causados por el herbicida y el crecimiento y rendimiento de tubérculo de plantas hijas. La ausencia de consistencia en la relación entre daño visual en la papa durante la producción de la planta madre y efectos adversos en el crecimiento y rendimiento de plantas hijas el siguiente año desafían la utilidad del muestreo tradicional del cultivo como herramienta para predecir el riesgo de daño accidental por herbicidas cerca de áreas para la producción de semilla de papa.
\end{abstract}

Potato accounts for $15 \%$ of total US vegetable sales, more than any other vegetable (USDA-ERS 2016). In 2014, the national potato crop was valued at $\$ 3.66$ billion from production on about 425,000 ha in over 30 states. Wisconsin ranked third in overall potato production, with a crop valued at $\$ 274$ million and produced on 25,900 ha (USDA-NASS 2015). When factoring in processing, potato production is a strong contributor to Wisconsin's rural economy, representing $\$ 349$ million in annual economic activity and accounting for 2,770 jobs (Arledge-Keene and Mitchell 2010).

This value, however, is dependent upon consistent potato crop quantity and quality. As early as 1985 , researchers began documenting negative effects of mother potato plant exposure to simulated off-target herbicides on daughter tuber growth and yield. Worthington (1985) reported that exposure of mother plants to glyphosate at $18 \mathrm{~g}$ ae ha ${ }^{-1}$ reduced daughter tuber emergence and resulted in malformed shoots. Potato yield response to glyphosate herbicide has been a subject of renewed research interest in recent years in response to widespread adoption of glyphosate-resistant agronomic crops near potato production. Hutchinson et al. (2014) evaluated the effect of several glyphosate rates applied to potato at 10 - to $15-\mathrm{cm}$ plant height, stolon hooking, tuber initiation, and mid-bulking. While glyphosate applications at mid-bulking caused the least foliar

DOI: $10.1017 /$ wet.2016.6

*Professor, Senior Outreach Specialist, and Senior Research Specialist, Department of Horticulture, University of Wisconsin, Madison, WI 53706. Corresponding author's E-mail: Colquhoun@wisc.edu 
injury, they had a greater impact on daughter tuber emergence and growth than the other application timings. Daughter tubers derived from mother plants that were treated with glyphosate at mid-bulking had emergence rates as low as 30\% of the emergence rate of daughter tubers of untreated plants, and displayed characteristic symptoms of glyphosate exposure such as chlorotic growing points. The authors pointed out that the discrepancy between the lack of visual foliar injury or tuber malformation in the mother plant and the poor emergence and appearance of injury in the daughter plants makes scouting of such situations a challenge in commercial production.

Such research was not limited to glyphosate. Wall (1994) investigated the impact of simulated drift of dicamba, clopyralid, and tribenuron on in-season mother plant injury and yield and daughter tuber growth the following season. While no tuber malformations were observed in the simulated drift year, marketable yields were reduced up to $75 \%$. In the following season, daughter plant injury was observed in 2 out of 3 study years, but tuber yields were unaffected.

While several studies have explored the relationship between simulated exposure to off-target herbicides and in-season crop growth in potato (Eberlein et al. 1997; Felix et al. 2011; Olszyk et al. 2010), few have investigated the relationship between mother plant exposure and daughter tuber growth in relation to herbicides other than glyphosate. Moreover, relatively new herbicides, such as topramezone and fluthiacet, are active at very low doses, but their off-target crop risks and effect on subsequent potato seed growth are unknown. The risk of off-target herbicide exposure through tank contamination is particularly relevant for producers who contract pesticide application from commercial applicators whose spray equipment is used for a broad range of crops, as many producers in Wisconsin do. The goal of this research was to determine the effect of simulated off-target herbicide exposure on mother potato plants (injury, yield, and quality) and their daughter potato plants (injury, yield, and quality) grown the following season. Herbicides evaluated here are commonly used in Wisconsin corn, soybean, and small grain fields, as well as pastures.

\section{Materials and Methods}

Two-year studies were initiated in 2013 and 2014 at the University of Wisconsin Hancock Agricultural Research Station. Mother potato plants were grown in the first year of each study, and the resulting daughter tubers were planted and grown in the second year. Soil type was a Plainfield loamy sand (sandy, mixed, mesic Typic Udipsamment) with $0.8 \%$ organic matter and a $\mathrm{pH}$ of 6.5 . Experiments were arranged in a randomized complete block design with four replications, with the identity of the replicates maintained for the duration of each twoyear study. In the mother plant production year, individual plots measured 6.1 by $3.7 \mathrm{~m}$, with four potato rows spaced $76 \mathrm{~cm}$ apart. In the daughter plant year, individual plots were $6.1 \mathrm{~m}$ long and consisted of a single row. The mother plants were planted on April 30, 2013 and May 5, 2014 and harvested on September 11, 2013 and September 18, 2014. The daughter plants were planted on May 5, 2014 and April 22, 2015 and harvested on September 17, 2014 and September 22, 2015.

The herbicides listed in Table 1 were applied with a tractor-mounted air pressure sprayer calibrated to deliver $187 \mathrm{~L} \mathrm{ha}^{-1}$ at $186 \mathrm{kPa}$ with Teejet ${ }^{\circledR}$ XR8003VS nozzle tips (Spraying Systems Co., P.O. Box 7900, Wheaton, IL 60187). Simulated off-target herbicide rates were selected based on $1 \%$ of the local commercial use rate in nearby agronomic crops. The herbicides were applied when tuber initiation was observed by digging potato hills in guard rows during crop scouting. Adjuvants were included as directed by the herbicide product label with concentrations adjusted to reflect $1 \%$ of the standard commercial application rates. The tuber initiation growth stage was chosen for herbicide application as it aligned with when applications would occur on nearby agronomic crops, such as corn (Zea mays L.), soybean (Glycine max [L.] Merr.), and small grains. Two additional glyphosate rates were included that were within the range used in related studies (Hutchinson et al. 2014). All plants were sprayed with $S$-metolachlor $\left(1.1 \mathrm{~kg}\right.$ ai ha $\left.{ }^{-1}\right)$ and metribuzin $\left(0.56 \mathrm{~kg}_{\text {ai ha }}{ }^{-1}\right)$ to control weeds. All other production practices, including fertilizer application, insect and disease management, and tuber storage between production seasons followed typical commercial practices (Colquhoun et al. 2016).

Data collection in the mother plant production year included visual estimation of potato foliar injury and tuber yield and quality. Foliar injury was estimated on a scale of $0 \%$ to $100 \%$, where $100 \%$ represents plant death. Tubers were harvested at crop maturity from one of the two center rows of each 
Table 1. Sources of herbicide used for studies in Hancock, WI from 2013 to 2015.

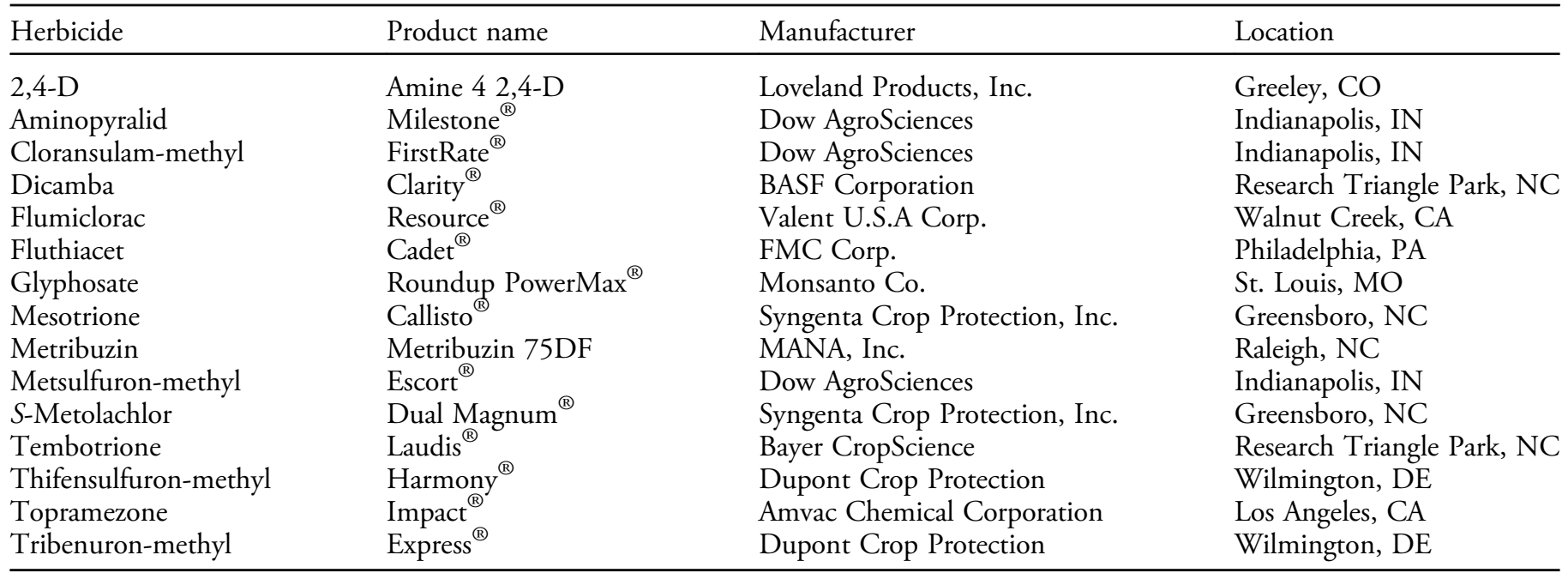

plot and graded according to US Department of Agriculture Agricultural Marketing Service standards (USDA-AMS 2013). Tuber specific gravity, a measure of tuber density that reflects potato processing quality, was determined using the water displacement method described by Dean and Thornton (1992). Data collection in the daughter plant year included the same parameters as described for the mother plant production year, plus stand density and affected plant incidence. The additional parameters were evaluated by counting the number of emerged plants and the number of injured plants in the rows that would be harvested. Data were subjected to ANOVA to determine if there was a year-by-variety interaction using PROC GLM in Statistical Analysis Software (SAS Institute Inc., Cary, NC 27513). An interaction was observed, and therefore data were analyzed and presented by crop and year. Means were separated using Fisher's LSD at $P=0.05$.

\section{Results and Discussion}

Mother Potato Plant Growth. Potato foliar injury was $10 \%$ by $5 \mathrm{~d}$ after treatment (DAT) where mesotrione was applied, and remained in that range through 20 DAT. Injury consisted primarily of bleached and stunted new foliage. Dicamba injury was $17 \%$ by 13 DAT, and remained high through the 29-d evaluation period. Similar injury severity was observed where aminopyralid was applied.
Symptomology for both herbicides consisted of severely cupped leaves at the meristematic stem ends. Mother potato plant foliar injury was $5 \%$ or less in all other treatments (Table 2). A higher incidence of injury was observed in 2014 than in 2013. We hypothesize that this may be due to environmental conditions near the time of herbicide application in 2014 that favored uptake and translocation, combined with subtle differences in the transition in sinks from the vegetative foliage to the initiating tubers among individual potato plants. Foliar injury patterns from dicamba and aminopyralid in 2014 were similar to those in 2013, with minimal early injury followed by $12 \%$ to $17 \%$ injury 12 DAT that persisted for the remainder of the 28-d evaluation period. Injury from mesotrione 4 DAT was $11 \%$, but dissipated to $1 \%$ by 28 DAT. In contrast to 2013, potato plant injury caused by the acetolactate synthase-inhibiting herbicides cloransulam-methyl, tribenuron-methyl, and metsulfuron-methyl was $10 \%$ or more at all evaluation times and was as high as $34 \%$ in some cases (Table 3).

Mother Potato Plant Yield. In 2013, minimal crop injury resulted in no differences in yield between the non-treated potato plants and the potato plants receiving herbicide treatments. Even where potato plant foliar injury from dicamba and aminopyralid persisted 29 DAT, no impact on potato yield or quality (assessed by cull tuber yield) was observed relative to non-treated potato plants (Table 4). In general, much greater yield response to herbicides 
Table 2. Visual estimation of mother potato plant foliar injury in 2013 in Hancock, Wisconsin.

\begin{tabular}{|c|c|c|c|c|c|c|}
\hline \multirow[b]{2}{*}{ Treatment } & \multirow[b]{2}{*}{ Herbicide rate } & \multirow[b]{2}{*}{ Adjuvant rate } & \multicolumn{4}{|c|}{ Visual estimation of potato foliar injury } \\
\hline & & & $5 \mathrm{DAT}^{\mathrm{a}}$ & 13 DAT & 20 DAT & 29 DAT \\
\hline & $\mathrm{g}$ ai or ae ha ${ }^{-1}$ & $\mathrm{~g} \mathrm{ha}^{-1}$ or $\% \mathrm{v} / \mathrm{v}^{\mathrm{b}}$ & & 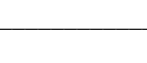 & & \\
\hline Non-treated & - & & 0 & 0 & 0 & 0 \\
\hline $2,4-\mathrm{D}$ & 5 & & 0 & 0 & 0 & 0 \\
\hline Dicamba + AMS & 6 & 28 & 0 & 17 & 25 & 16 \\
\hline Glyphosate + AMS & 38 & 76 & 3 & 1 & 0 & 0 \\
\hline Mesotrione $+\mathrm{AMS}+\mathrm{COC}^{\mathrm{a}}$ & 1 & $19+0.01$ & 10 & 12 & 11 & 1 \\
\hline Topramezone + AMS + MSO ${ }^{\mathrm{a}}$ & 0.2 & $19+0.01$ & 1 & 2 & 0 & 0 \\
\hline Tembotrione + AMS + MSO & 0.9 & $19+0.01$ & 5 & 1 & 2 & 0 \\
\hline Fluthiacet + AMS + NIS $^{\mathrm{a}}$ & 0.06 & $17+0.01$ & 0 & 0 & 0 & 0 \\
\hline Aminopyralid + NIS & 0.9 & 0.0025 & 0 & 14 & 21 & 18 \\
\hline Metsulfuron-methyl + NIS & 0.1 & 0.0025 & 0 & 0 & 0 & 0 \\
\hline
\end{tabular}

${ }^{\mathrm{a}}$ Abbreviations: AMS, ammonium sulfate; COC, crop oil concentrate; DAT, $\mathrm{d}$ after treatment; MSO, methylated seed oil; NIS, non-ionic surfactant.

${ }^{\mathrm{b}}$ AMS rates are in $\mathrm{g} \mathrm{ha}^{-1}$. COC, MSO, and NIS rates are in $\% \mathrm{v} / \mathrm{v}$.

was observed in 2014 compared to 2013. For example, in 2014 all herbicide treatments except topramezone reduced the yield of tubers weighing
113 to $169 \mathrm{~g}$ compared to non-treated potato plants. The yield of cull tubers, those malformed or blemished beyond marketability, was greatest among

Table 3. Visual estimation of mother potato plant foliar injury in 2014 in Hancock, Wisconsin.

\begin{tabular}{|c|c|c|c|c|c|c|}
\hline \multirow[b]{2}{*}{ Treatment } & \multirow[b]{2}{*}{ Herbicide rate } & \multirow[b]{2}{*}{ Adjuvant rate } & \multicolumn{4}{|c|}{ Visual estimation of potato foliar injury } \\
\hline & & & $4 \mathrm{DAT}^{\mathrm{a}}$ & 12 DAT & 19 DAT & $28 \mathrm{DAT}$ \\
\hline & g ai or ae $\mathrm{ha}^{-1}$ & $\mathrm{~g} \mathrm{ha}^{-1}$ or $\% \mathrm{v} / \mathrm{v}^{\mathrm{b}}$ & . & 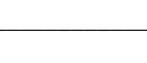 & & \\
\hline Non-treated & - & & 0 & 0 & 0 & 0 \\
\hline $2,4-\mathrm{D}$ & 5 & & 1 & 1 & 0 & 0 \\
\hline Dicamba + AMS & 6 & 28 & 2 & 12 & 15 & 17 \\
\hline Glyphosate + AMS & 38 & 76 & 8 & 7 & 6 & 3 \\
\hline Mesotrione $+\mathrm{AMS}+\mathrm{COC}^{\mathrm{a}}$ & 1 & $19+0.01$ & 11 & 24 & 6 & 1 \\
\hline Topramezone + AMS + $\mathrm{MSO}^{\mathrm{a}}$ & 0.2 & $19+0.01$ & 7 & 3 & 3 & 0 \\
\hline Tembotrione + AMS + MSO & 0.9 & $19+0.01$ & 4 & 4 & 2 & 0 \\
\hline Fluthiacet + AMS + NIS ${ }^{\mathrm{a}}$ & 0.06 & $17+0.01$ & 2 & 1 & 1 & 0 \\
\hline Aminopyralid + NIS & 0.9 & 0.0025 & 6 & 11 & 10 & 16 \\
\hline Metsulfuron-methyl + NIS & 0.1 & 0.0025 & 13 & 34 & 34 & 21 \\
\hline
\end{tabular}

a Abbreviations: AMS, ammonium sulfate; COC, crop oil concentrate; DAT, $\mathrm{d}$ after treatment; MSO, methylated seed oil; NIS, non-ionic surfactant.

b AMS rates are in $\mathrm{g} \mathrm{ha}^{-1}$. COC, MSO, and NIS rates are in $\% \mathrm{v} / \mathrm{v}$. 
Table 4. Mother potato plant tuber yield in 2013 in Hancock, Wisconsin.

\begin{tabular}{|c|c|c|c|c|c|c|c|c|c|c|c|}
\hline \multirow[b]{2}{*}{ Treatment } & \multirow[b]{2}{*}{ Herbicide rate } & \multirow[b]{2}{*}{ Adjuvant rate } & \multicolumn{9}{|c|}{ Potato tuber yield ${ }^{a}$} \\
\hline & & & $\bar{B} \operatorname{size}^{\mathrm{b}}$ & Cull & $57-112 \mathrm{~g}$ & $113-169 \mathrm{~g}$ & $170-282 \mathrm{~g}$ & $283-368 \mathrm{~g}$ & $369-454 \mathrm{~g}$ & $>454 \mathrm{~g}$ & $\overline{\text { Total }}$ \\
\hline & $\mathrm{g}$ ai or ae $\mathrm{ha}^{-1}$ & $\mathrm{~g} \mathrm{ha}^{-1}$ or $\% \mathrm{v} / \mathrm{v}^{\mathrm{c}}$ & & & & & $-\mathrm{kg}$ & & & & \\
\hline Non-treated & - & & 264 & 7,115 & 7,493 & 16,153 & 27,772 & 8,841 & 5,367 & 4,848 & 77,852 \\
\hline $2,4-\mathrm{D}$ & 5 & & 272 & 7,481 & 7,070 & 16,868 & 30,994 & 10,697 & 3,789 & 4,013 & 81,184 \\
\hline Dicamba + AMS & 6 & 28 & 128 & 6,139 & 8,330 & 14,644 & 30,897 & 13,374 & 3,793 & 5,590 & 82,896 \\
\hline Glyphosate + AMS ${ }^{\mathrm{d}}$ & 9 & 19 & 319 & 6,932 & 7,308 & 14,500 & 30,636 & 9,489 & 6,775 & 3,692 & 79,651 \\
\hline Glyphosate + AMS & 19 & 38 & 128 & 7,542 & 6,371 & 14,654 & 25,727 & 12,670 & 5,230 & 6,127 & 78,450 \\
\hline Glyphosate + AMS & 38 & 76 & 325 & 4,492 & 8,875 & 18,952 & 31,954 & 12,628 & 4,684 & 2,899 & 84,808 \\
\hline Mesotrione + AMS + COC & 1 & $19+0.01$ & 258 & 5,875 & 7,855 & 15,470 & 27,937 & 11,642 & 4,588 & 3,498 & 77,122 \\
\hline Topramezone + AMS + MSO & 0.2 & $19+0.01$ & 183 & 5,529 & 6,875 & 14,305 & 29,522 & 12,402 & 6,141 & 4,486 & 79,444 \\
\hline Tembotrione + AMS + MSO & 0.9 & $19+0.01$ & 201 & 6,830 & 6,844 & 15,777 & 31,594 & 13,000 & 4,541 & 4,728 & 83,516 \\
\hline Fluthiacet + AMS + NIS & 0.06 & $17+0.01$ & 177 & 6,769 & 6,838 & 14,783 & 33,135 & 10,530 & 7,698 & 3,124 & 83,054 \\
\hline Cloransulam-methyl + AMS + NIS & 0.4 & $22+0.0025$ & 283 & 9,371 & 8,802 & 16,496 & 26,963 & 10,882 & 5,899 & 5,861 & 84,556 \\
\hline Flumiclorac + COC & 0.6 & 16 & 463 & 7,033 & 7,639 & 15,716 & 31,094 & 11,296 & 6,133 & 3,498 & 82,873 \\
\hline Thifensulfuron-methyl + AMS + NIS & 0.04 & $22+0.0025$ & 289 & 4,919 & 7,139 & 17,850 & 34,316 & 10,764 & 5,493 & 3,838 & 84,607 \\
\hline Tribenuron-methyl + AMS + NIS & 0.2 & $22+0.0025$ & 293 & 8,721 & 7,999 & 16,199 & 32,067 & 11,908 & 3,446 & 2,765 & 83,398 \\
\hline Aminopyralid + NIS & 0.9 & 0.0025 & 185 & 5,550 & 10,705 & 18,637 & 29,171 & 8,538 & 4,125 & 1,813 & 78,722 \\
\hline Metsulfuron-methyl + NIS & 0.1 & 0.0025 & 232 & 6,891 & 8,178 & 15,866 & 29,272 & 10,615 & 6,161 & 2,496 & 79,712 \\
\hline
\end{tabular}

${ }^{a}$ Means within columns followed by the same letter are not significantly different according to Fisher's protected LSD test at $P \leq 0.05$. No significant differences within a column were observed when no letters are included.

${ }^{\mathrm{b}} \mathrm{B}$ size potatoes include those with a diameter of $4.4 \mathrm{~cm}$ or less.

${ }^{c}$ AMS rates are in $\mathrm{g} \mathrm{ha}^{-1}$. COC, MSO, and NIS rates are in $\% \mathrm{v} / \mathrm{v}$.

d Abbreviations: AMS, ammonium sulfate; COC, crop oil concentrate; MSO, methylated seed oil; NIS, non-ionic surfactant. 
Table 5. Mother potato plant tuber yield in 2014 in Hancock, Wisconsin.

\begin{tabular}{|c|c|c|c|c|c|c|c|c|c|c|c|}
\hline \multirow[b]{2}{*}{ Treatment } & \multirow{2}{*}{$\begin{array}{l}\text { Herbicide } \\
\text { rate }\end{array}$} & \multirow{2}{*}{$\begin{array}{l}\text { Adjuvant } \\
\text { rate }\end{array}$} & \multicolumn{9}{|c|}{ Potato tuber yield ${ }^{a}$} \\
\hline & & & $\mathrm{B} \operatorname{size}^{\mathrm{b}}$ & Cull & $57-112 \mathrm{~g}$ & $113-169 \mathrm{~g}$ & $170-282 \mathrm{~g}$ & $283-368 \mathrm{~g}$ & $369-454 \mathrm{~g}$ & $>454 \mathrm{~g}$ & Total \\
\hline & $\mathrm{g}$ ai or ae $\mathrm{ha}^{-1}$ & $\mathrm{~g} \mathrm{ha}^{-1}$ or $\% \mathrm{v} / \mathrm{v}^{\mathrm{c}}$ & & & & & $\mathrm{kg} \mathrm{ha}^{-1}$ & & & & \\
\hline Non-treated & - & & $1,464 \mathrm{bc}$ & 3,822 ef & $10,197 \mathrm{a}$ & $17,559 \mathrm{a}$ & $22,481 \mathrm{ab}$ & $4,736 \mathrm{bc}$ & $2,937 \mathrm{bc}$ & $1,637 \mathrm{~b}-\mathrm{e}$ & $65,253 \mathrm{a}$ \\
\hline $2,4-D$ & 5 & & $1,400 \mathrm{bcd}$ & 5,478 ef & $9,300 \mathrm{ab}$ & $14,097 \mathrm{~b}$ & $20,690 \mathrm{abc}$ & $6,406 \mathrm{bcd}$ & $1,958 \mathrm{bcd}$ & 766 def & $61,071 \mathrm{a}$ \\
\hline Dicamba + AMS & 6 & 28 & $1,260 \mathrm{bcd}$ & $4,513 \mathrm{ef}$ & $9,072 \mathrm{abc}$ & $13,644 \mathrm{~b}$ & $20,419 \mathrm{abc}$ & $7,501 \mathrm{abc}$ & $3,450 \mathrm{ab}$ & $4,370 \mathrm{ab}$ & $64,560 \mathrm{a}$ \\
\hline Glyphosate + AMS ${ }^{\mathrm{d}}$ & 9 & 19 & 1,329 bcd & 7,013 def & $7,328 \mathrm{~b}-\mathrm{e}$ & $12,672 \mathrm{bc}$ & $19,139 \mathrm{bc}$ & $7,059 \mathrm{abc}$ & $3,690 \mathrm{ab}$ & 2,369 a-e & $60,748 \mathrm{a}$ \\
\hline Glyphosate + AMS & 19 & 38 & $1,719 \mathrm{~b}$ & 3,740 ef & 8,469 a-d & $13,333 \mathrm{bc}$ & $23,300 \mathrm{ab}$ & $7,520 \mathrm{abc}$ & $2,346 \mathrm{bcd}$ & $910 \mathrm{c}-\mathrm{f}$ & $61,858 \mathrm{a}$ \\
\hline Glyphosate + AMS & 38 & 76 & $1,003 \mathrm{~cd}$ & 5,509 ef & 7,208 b-e & $11,727 \mathrm{bc}$ & $23,233 \mathrm{ab}$ & $9,603 \mathrm{a}$ & $5,702 \mathrm{a}$ & 2,507 a-e & $67,570 \mathrm{a}$ \\
\hline Mesotrione + AMS + COC & 1 & $19+0.01$ & $939 \mathrm{~cd}$ & 6,190 def & $8,851 \mathrm{abc}$ & $13,679 \mathrm{~b}$ & $16,712 \mathrm{c}$ & $8,162 \mathrm{ab}$ & $3,287 \mathrm{ab}$ & $3,490 \mathrm{abc}$ & $62,015 \mathrm{a}$ \\
\hline Topramezone + AMS + MSO & 0.2 & $19+0.01$ & $1,067 \mathrm{bcd}$ & 4,543 ef & 7,145 b-e & $14,551 \mathrm{ab}$ & $24,995 \mathrm{a}$ & $8,311 \mathrm{ab}$ & $4,135 \mathrm{ab}$ & $2,913 \mathrm{a}-\mathrm{d}$ & $68,050 \mathrm{a}$ \\
\hline Tembotrione + AMS + MSO & 0.9 & $19+0.01$ & $1,084 \mathrm{bcd}$ & $3,334 \mathrm{f}$ & 6,922 cde & $11,912 \mathrm{bc}$ & $21,466 \mathrm{abc}$ & $10,080 \mathrm{a}$ & $4,580 \mathrm{ab}$ & 5,636 a & $65,253 \mathrm{a}$ \\
\hline Fluthiacet + AMS + NIS & 0.06 & $17+0.01$ & $1,230 \mathrm{bcd}$ & 3,893 ef & 8,290 a-d & $13,199 \mathrm{bc}$ & $22,562 \mathrm{ab}$ & $7,280 \mathrm{abc}$ & $3,746 \mathrm{ab}$ & $4,582 \mathrm{ab}$ & $65,926 \mathrm{a}$ \\
\hline Cloransulam-methyl + AMS + NIS & 0.4 & $22+0.0025$ & $814 \mathrm{~d}$ & $14,616 \mathrm{bc}$ & $6,052 \mathrm{e}$ & $7,151 \mathrm{de}$ & $11,060 \mathrm{~d}$ & $4,463 \mathrm{c}$ & $2,555 \mathrm{bcd}$ & 2,848 a-e & $50,283 \mathrm{bc}$ \\
\hline Flumiclorac + COC & 0.6 & 16 & $882 \mathrm{~cd}$ & 3,791 ef & 8,227 a-e & $13,488 \mathrm{bc}$ & $23,526 \mathrm{ab}$ & $7,813 \mathrm{abc}$ & $3,942 \mathrm{ab}$ & 2,980 a-d & $65,342 \mathrm{a}$ \\
\hline Thifensulfuron-methyl + AMS + NIS & 0.04 & $22+0.0025$ & $938 \mathrm{~cd}$ & $12,329 \mathrm{~cd}$ & 8,111 a-e & $10,107 \mathrm{~cd}$ & $19,690 \mathrm{bc}$ & $7,539 \mathrm{abc}$ & $3,098 \mathrm{ab}$ & 2,385 a-e & 64,838 a \\
\hline Tribenuron-methyl + AMS + NIS & 0.2 & $22+0.0025$ & $1,355 \mathrm{bcd}$ & $45,057 \mathrm{a}$ & 6,438 de & $6,574 \mathrm{e}$ & $4,738 \mathrm{e}$ & $375 \mathrm{~d}$ & $366 \mathrm{~cd}$ & $0 \mathrm{f}$ & $65,218 \mathrm{a}$ \\
\hline Aminopyralid + NIS & 0.9 & 0.0025 & $1,509 \mathrm{bc}$ & 9,951 cde & $9,898 \mathrm{a}$ & $12,648 \mathrm{bc}$ & $16,388 \mathrm{c}$ & $4,941 \mathrm{bc}$ & $2,222 \mathrm{bcd}$ & 484 ef & $59,022 \mathrm{ab}$ \\
\hline Metsulfuron-methyl + NIS & 0.1 & 0.0025 & $3,068 \mathrm{a}$ & $20,196 \mathrm{~b}$ & $9,942 \mathrm{a}$ & $6,777 \mathrm{de}$ & $5,054 \mathrm{e}$ & $273 \mathrm{~d}$ & $0 \mathrm{~d}$ & $0 \mathrm{f}$ & $45,620 \mathrm{c}$ \\
\hline
\end{tabular}

a Means within columns followed by the same letter are not significantly different according to Fisher's protected LSD test at $P \leq 0.05$. No significant differences within a column were observed when no letters are included.

b $\mathrm{B}$ size potatoes include those with a diameter of $4.4 \mathrm{~cm}$ or less.

${ }^{c}$ AMS rates are in $\mathrm{g} \mathrm{ha}^{-1}$. COC, MSO, and NIS rates are in $\% \mathrm{v} / \mathrm{v}$.

d Abbreviations: AMS, ammonium sulfate; COC, crop oil concentrate; MSO, methylated seed oil; NIS, non-ionic surfactant. 
Table 6. Daughter potato plant stand density, number of affected plants and visual estimation of foliar injury in 2014, the year after herbicide exposure, in Hancock, Wisconsin.

\begin{tabular}{|c|c|c|c|c|c|c|c|c|c|}
\hline \multirow[b]{2}{*}{ Treatment } & \multirow[b]{2}{*}{ Herbicide rate } & \multirow[b]{2}{*}{ Adjuvant rate } & \multicolumn{2}{|c|}{ Stand density } & \multicolumn{2}{|c|}{ Affected plants } & \multicolumn{3}{|c|}{ Visual estimation of foliar injury } \\
\hline & & & $30 \mathrm{DAP}^{\mathrm{a}, \mathrm{b}}$ & 35 DAP & $42 \mathrm{DAP}$ & $48 \mathrm{DAP}$ & 36 DAP & $42 \mathrm{DAP}$ & 48 DAP \\
\hline & $\mathrm{g}$ ai or ae $\mathrm{ha}^{-1}$ & $\mathrm{~g} \mathrm{ha}{ }^{-1}$ or $\% \mathrm{v} / \mathrm{v}^{\mathrm{c}}$ & - & plants & $a^{-1}$ & & & $\%$ & \\
\hline Non-treated & - & & $24,757 \mathrm{ab}$ & 34,983 & 3,229 & 0 & 1 & 8 & 0 \\
\hline $2,4-\mathrm{D}$ & 5 & & $31,933 \mathrm{a}$ & 34,983 & 0 & 718 & 0 & 0 & 1 \\
\hline Dicamba + AMS & 6 & 28 & $28,345 \mathrm{ab}$ & 35,521 & 2,691 & 2,153 & 8 & 11 & 1 \\
\hline Glyphosate $+\mathrm{AMS}^{\mathrm{b}}$ & 9 & 19 & $29,242 \mathrm{ab}$ & 34,086 & 538 & 538 & 0 & 10 & 1 \\
\hline Glyphosate + AMS & 19 & 38 & $22,425 \mathrm{~b}$ & 33,727 & 359 & 359 & 3 & 4 & 1 \\
\hline Glyphosate + AMS & 38 & 76 & $26,013 \mathrm{ab}$ & 35,521 & 1,435 & 0 & 0 & 5 & 0 \\
\hline Mesotrione + AMS + COC & 1 & $19+0.01$ & $28,345 \mathrm{ab}$ & 35,521 & 718 & 1,076 & 9 & 4 & 1 \\
\hline Topramezone + AMS + MSO & 0.2 & $19+0.01$ & $25,116 \mathrm{ab}$ & 35,521 & 1,794 & 538 & 2 & 4 & 1 \\
\hline Tembotrione + AMS + MSO & 0.9 & $19+0.01$ & $29,601 \mathrm{ab}$ & 34,983 & 179 & 0 & 0 & 1 & 0 \\
\hline Fluthiacet + AMS + NIS & 0.06 & $17+0.01$ & $22,425 \mathrm{~b}$ & 34,624 & 5,203 & 3,229 & 4 & 10 & 2 \\
\hline Cloransulam-methyl + AMS + NIS & 0.4 & $22+0.0025$ & $25,654 \mathrm{ab}$ & 35,521 & 0 & 0 & 3 & 0 & 0 \\
\hline Flumiclorac + COC & 0.6 & 16 & $30,498 \mathrm{ab}$ & 35,880 & 0 & 0 & 0 & 0 & 0 \\
\hline Thifensulfuron-methyl + AMS + NIS & 0.04 & $22+0.0025$ & $25,654 \mathrm{ab}$ & 35,521 & 2,332 & 3,050 & 5 & 8 & 2 \\
\hline Tribenuron-methyl + AMS + NIS & 0.2 & $22+0.0025$ & $31,036 \mathrm{ab}$ & 34,983 & 179 & 0 & 0 & 6 & 0 \\
\hline Aminopyralid + NIS & 0.9 & 0.0025 & $30,498 \mathrm{ab}$ & 36,418 & 5,561 & 718 & 2 & 11 & 1 \\
\hline Metsulfuron-methyl + NIS & 0.1 & 0.0025 & $28,704 \mathrm{ab}$ & 34,086 & 179 & 1,076 & 2 & 3 & 1 \\
\hline
\end{tabular}

a Stand density and affected plant means within columns followed by the same letter are not significantly different according to Fisher's protected LSD test at $P \leq 0.05$. No significant differences within a column were observed when no letters are included.

b Abbreviations: AMS, ammonium sulfate; COC, crop oil concentrate; DAP, d after planting; MSO, methylated seed oil; NIS, non-ionic surfactant.

${ }^{c}$ AMS rates are in $\mathrm{g} \mathrm{ha}^{-1}$. COC, MSO, and NIS rates are in $\% \mathrm{v} / \mathrm{v}$. 
Table 7. Daughter potato plant stand density, number of affected plants and visual estimation of foliar injury in 2015, the year after herbicide exposure, in Hancock, Wisconsin.

\begin{tabular}{|c|c|c|c|c|c|c|c|c|c|}
\hline \multirow[b]{2}{*}{ Treatment } & \multirow[b]{2}{*}{ Herbicide rate } & \multirow[b]{2}{*}{ Adjuvant rate } & \multicolumn{2}{|c|}{ Stand density } & \multicolumn{2}{|c|}{ Affected plants } & \multicolumn{3}{|c|}{ Visual estimation of foliar injury } \\
\hline & & & $35 \mathrm{DAP}^{\mathrm{a}, \mathrm{b}}$ & 43 DAP & 49 DAP & 56 DAP & 43 DAP & 49 DAP & 56 DAP \\
\hline & g ai or ae $\mathrm{ha}^{-1}$ & $\mathrm{~g} \mathrm{ha}^{-1}$ or $\% \mathrm{v} / \mathrm{v}^{\mathrm{c}}$ & 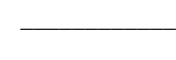 & plan & $s \mathrm{ha}^{-1}$ & - & & $\%$ & \\
\hline Non-treated & - & & $29,601 \mathrm{ab}$ & $32,292 \mathrm{~b}$ & $179 \mathrm{~cd}$ & $1,794 \mathrm{~cd}$ & 1 & 1 & 1 \\
\hline $2,4-\mathrm{D}$ & 5 & & $32,830 \mathrm{a}$ & $34,983 \mathrm{ab}$ & $0 \mathrm{~d}$ & $0 \mathrm{~d}$ & 6 & 0 & 0 \\
\hline Dicamba + AMS & 6 & 28 & $28,704 \mathrm{ab}$ & $33,727 \mathrm{ab}$ & $20,631 \mathrm{a}$ & $10,226 \mathrm{ab}$ & 50 & 37 & 7 \\
\hline Glyphosate + $\mathrm{AMS}^{\mathrm{b}}$ & 9 & 19 & $29,242 \mathrm{ab}$ & $34,983 \mathrm{ab}$ & $0 \mathrm{~d}$ & $718 \mathrm{~d}$ & 5 & 0 & 0 \\
\hline Glyphosate + AMS & 19 & 38 & $31,036 \mathrm{ab}$ & $34,983 \mathrm{ab}$ & $538 \mathrm{~cd}$ & $359 \mathrm{~d}$ & 16 & 2 & 0 \\
\hline Glyphosate + AMS & 38 & 76 & $23,860 \mathrm{bc}$ & $31,933 \mathrm{~b}$ & $6,279 \mathrm{~b}$ & $4,664 \mathrm{bc}$ & 18 & 30 & 5 \\
\hline Mesotrione + AMS + COC & 1 & $19+0.01$ & $34,624 \mathrm{a}$ & $35,880 \mathrm{a}$ & $538 \mathrm{~cd}$ & $718 \mathrm{~d}$ & 10 & 1 & 0 \\
\hline Topramezone + AMS + MSO & 0.2 & $19+0.01$ & 33,189 a & $34,983 \mathrm{ab}$ & $0 \mathrm{~d}$ & $0 \mathrm{~d}$ & 5 & 0 & 0 \\
\hline Tembotrione + AMS + MSO & 0.9 & $19+0.01$ & $34,086 \mathrm{a}$ & $34,983 \mathrm{ab}$ & $538 \mathrm{~cd}$ & $0 \mathrm{~d}$ & 4 & 1 & 0 \\
\hline Fluthiacet + AMS + NIS & 0.06 & $17+0.01$ & 33,727 a & $34,983 \mathrm{ab}$ & $0 \mathrm{~d}$ & $0 \mathrm{~d}$ & 6 & 0 & 0 \\
\hline Cloransulam-methyl + AMS + NIS & 0.4 & $22+0.0025$ & $31,933 \mathrm{a}$ & $35,880 \mathrm{a}$ & $718 \mathrm{bcd}$ & $0 \mathrm{~d}$ & 8 & 3 & 0 \\
\hline Flumiclorac + COC & 0.6 & 16 & $29,242 \mathrm{ab}$ & $33,727 \mathrm{ab}$ & $718 \mathrm{bcd}$ & $718 \mathrm{~d}$ & 5 & 2 & 0 \\
\hline Thifensulfuron-methyl + AMS + NIS & 0.04 & $22+0.0025$ & $30,139 \mathrm{ab}$ & $34,983 \mathrm{ab}$ & $3,947 \mathrm{bc}$ & $1,615 \mathrm{~d}$ & 8 & 5 & 1 \\
\hline Tribenuron-methyl + AMS + NIS & 0.2 & $22+0.0025$ & $31,395 \mathrm{ab}$ & $34,624 \mathrm{ab}$ & $0 \mathrm{~d}$ & $0 \mathrm{~d}$ & 3 & 0 & 0 \\
\hline Aminopyralid + NIS & 0.9 & 0.0025 & $19,375 \mathrm{c}$ & $25,654 \mathrm{c}$ & $21,887 \mathrm{a}$ & $17,043 \mathrm{a}$ & 58 & 54 & 35 \\
\hline Metsulfuron-methyl + NIS & 0.1 & 0.0025 & $26,910 \mathrm{abc}$ & $33,189 \mathrm{ab}$ & $1,615 \mathrm{bcd}$ & $538 \mathrm{~d}$ & 10 & 6 & 0 \\
\hline
\end{tabular}

${ }^{a}$ Stand density and affected plant means within columns followed by the same letter are not significantly different according to Fisher's protected LSD test at $P \leq 0.05$. No significant differences within a column were observed when no letters are included.

b Abbreviations: AMS, ammonium sulfate; DAP, d after planting; COC, crop oil concentrate; MSO, methylated seed oil; NIS, non-ionic surfactant.

${ }^{c}$ AMS rates are in $\mathrm{g} \mathrm{ha}^{-1}$. COC, MSO, and NIS rates are in $\% \mathrm{v} / \mathrm{v}$. 
Table 8. Daughter potato plant tuber yield in 2014, the year after herbicide exposure, in Hancock, Wisconsin.

Potato tuber yield ${ }^{a}$

\begin{tabular}{|c|c|c|c|c|c|c|c|c|c|c|c|}
\hline Treatment & Herbicide rate & Adjuvant rate & $\bar{B}$ size $^{b}$ & Cull & $57-112 \mathrm{~g}$ & $113-169 \mathrm{~g}$ & $170-282 \mathrm{~g}$ & $283-368 \mathrm{~g}$ & $369-454 \mathrm{~g}$ & $>454 \mathrm{~g}$ & Total \\
\hline & $\mathrm{g}$ ai or ae ha ${ }^{-1}$ & $\mathrm{~g} \mathrm{ha}^{-1}$ or $\% \mathrm{v} / \mathrm{v}^{\mathrm{c}}$ & & & & & $-\mathrm{kg} \mathrm{ha}^{-1}$ & & & & \\
\hline Non-treated & - & & 1,168 & 7,694 & 6,991 & 10,173 & 16,686 & $10,564 \mathrm{ab}$ & 7,196 & 4,297 & 57,826 \\
\hline $2,4-\mathrm{D}$ & 5 & & 945 & 11,055 & 6,580 & 9,612 & 21,563 & $10,007 \mathrm{ab}$ & 4,896 & 2,913 & 58,013 \\
\hline Dicamba + AMS & 6 & 28 & 1,159 & 9,841 & 6,342 & 9,950 & 19,189 & $6,184 c$ & 3,956 & 3,861 & 51,075 \\
\hline Glyphosate + $A M S^{d}$ & 9 & 19 & 954 & 8,315 & 7,730 & 11,392 & 18,591 & $8,754 \mathrm{abc}$ & 3,002 & 3,844 & 55,233 \\
\hline Glyphosate + AMS & 19 & 38 & 1,271 & 5,188 & 5,562 & 10,971 & 21,410 & $9,048 \mathrm{abc}$ & 5,648 & 2,234 & 56,396 \\
\hline Glyphosate + AMS & 38 & 76 & 993 & 10,055 & 7,312 & 11,626 & 21,420 & $10,523 \mathrm{ab}$ & 6,574 & 2,580 & 61,719 \\
\hline Mesotrione + AMS + COC & 1 & $19+0.01$ & 435 & 13,935 & 5,066 & 9,624 & 16,280 & $8,064 \mathrm{bc}$ & 6,828 & 3,851 & 50,613 \\
\hline Topramezone + AMS + MSO & 0.2 & $19+0.01$ & 1,093 & 7,798 & 5,689 & 10,510 & 17,422 & $10,533 \mathrm{ab}$ & 6,194 & 3,431 & 55,037 \\
\hline one + AMS + MSO & 0.9 & $19+0.01$ & 929 & 6,808 & 5,859 & 10,500 & 20,007 & $8,572 a b c$ & 5,809 & 4,763 & 56,717 \\
\hline Fluthiacet + AMS + NIS & 0.06 & $17+0.01$ & 968 & 12,965 & 5,775 & 9,633 & 22,052 & $10,985 \mathrm{ab}$ & 4,860 & 4,268 & 59,101 \\
\hline Cloransulam-methyl + AMS + NIS & 0.4 & $22+0.0025$ & 1,119 & 10,786 & 6,340 & 10,988 & 19,901 & & 4,714 & 2,753 & 52,394 \\
\hline Flumiclorac + COC & 0.6 & 16 & 810 & 10,218 & 6,334 & 8,713 & 21,524 & $10,603 \mathrm{ab}$ & 7,174 & 6,502 & 62,104 \\
\hline Thifensulfuron-methyl + AMS + NIS & 0.04 & $22+0.0025$ & 936 & 7,671 & 6,005 & 11,251 & 17,775 & $10,585 \mathrm{ab}$ & 4,897 & 2,624 & 54,330 \\
\hline Tribenuron-methyl + AMS + NIS & 0.2 & $22+0.0025$ & 1,410 & 10,250 & 7,348 & 10,679 & 20,351 & $6,195 c$ & 4,642 & 2,446 & 53,429 \\
\hline alid + NIS & 0.9 & 0.0025 & 1,155 & 7,814 & 7,615 & 11,124 & 21,954 & $9,070 \mathrm{abc}$ & 3,654 & 1,755 & 56,654 \\
\hline Metsulfuron-methyl + NIS & 0.1 & 0.0025 & 913 & 10,736 & 5,579 & 8,567 & 20,278 & $12,246 \mathrm{a}$ & 7,695 & 6,535 & 62,175 \\
\hline
\end{tabular}

${ }^{a}$ Means within columns followed by the same letter are not significantly different according to Fisher's protected LSD test at $P \leq 0.05$. No significant differences within a column were observed when no letters are included.

${ }^{\mathrm{b}} \mathrm{B}$ size potatoes include those with a diameter of $4.4 \mathrm{~cm}$ or less.

${ }^{c}$ AMS rates are in $\mathrm{g} \mathrm{ha}^{-1}$. COC, MSO, and NIS rates are in $\% \mathrm{v} / \mathrm{v}$.

${ }^{\mathrm{d}}$ Abbreviations: AMS, ammonium sulfate; COC, crop oil concentrate; MSO, methylated seed oil; NIS, non-ionic surfactant. 
Table 9. Daughter potato plant tuber yield in 2015, the year after herbicide exposure, in Hancock, Wisconsin.

\begin{tabular}{|c|c|c|c|c|c|c|c|c|c|c|c|}
\hline \multirow[b]{2}{*}{ Treatment } & \multirow[b]{2}{*}{ Herbicide rate } & \multirow[b]{2}{*}{ Adjuvant rate } & \multicolumn{9}{|c|}{ Potato tuber yield ${ }^{a}$} \\
\hline & & & B size $^{b}$ & Cull & $57-112 \mathrm{~g}$ & $113-169 \mathrm{~g}$ & $170-282 \mathrm{~g}$ & $283-368 \mathrm{~g}$ & $369-454 \mathrm{~g}$ & $>454 \mathrm{~g}$ & Total \\
\hline & $\mathrm{g}$ ai or ae ha ${ }^{-}$ & $\mathrm{g} \mathrm{ha}^{-1}$ or $\% \mathrm{v} / \mathrm{v}$ & & & & & $\mathrm{kg} \mathrm{h}$ & & & & \\
\hline Non-treated & - & & 519 & 4,627 & $8,382 \mathrm{bcd}$ & $18,846 \mathrm{ab}$ & 31,134 & 10,334 & 5,907 & 4,443 & $84,432 \mathrm{ab}$ \\
\hline $2,4-\mathrm{D}$ & 5 & & 697 & 7,906 & $8,620 \mathrm{bc}$ & 17,208 a-e & 28,475 & 12,039 & 3,023 & 5,210 & $83,792 \mathrm{ab}$ \\
\hline Dicamba + AMS & 6 & 28 & 632 & 5,309 & $9,505 \mathrm{ab}$ & 14,860 a-f & 24,975 & 9,643 & 4,996 & 2,618 & $72,686 \mathrm{bc}$ \\
\hline Glyphosate + AMS & 9 & 19 & 699 & 3,690 & $11,052 \mathrm{a}$ & $18,054 \mathrm{a}-\mathrm{d}$ & 25,421 & 10,591 & 2,851 & 3,687 & $76,325 \mathrm{ab}$ \\
\hline Glyphosate + AMS & 19 & 38 & 614 & 6,226 & $8,514 \mathrm{bc}$ & $18,441 \mathrm{abc}$ & 28,436 & 13,159 & 4,348 & 5,153 & $85,811 \mathrm{a}$ \\
\hline Glyphosate + AMS & 38 & 76 & 654 & 5,125 & 7,589 b-e & $13,423 \mathrm{def}$ & 27,224 & 11,262 & 3,120 & 6,268 & $75,182 \mathrm{ab}$ \\
\hline Mesotrione + AMS + COC & 1 & $19+0.01$ & 567 & 6,051 & $7,763 \mathrm{bcd}$ & $13,543 \mathrm{c}-\mathrm{f}$ & 27,440 & 10,493 & 7,310 & 8,032 & $81,925 \mathrm{ab}$ \\
\hline Topramezone + AMS + MSO & 0.2 & $19+0.01$ & 493 & 8,020 & $6,180 \mathrm{de}$ & $14,463 \mathrm{~b}-\mathrm{f}$ & 27,458 & 13,441 & 6,069 & 5,688 & $82,152 \mathrm{ab}$ \\
\hline$+\mathrm{MSO}$ & 0.9 & $19+0.01$ & 615 & 5,899 & 7,010 cde & 16,445 a-e & 29,730 & 11,425 & 5,015 & 5,228 & $81,543 \mathrm{ab}$ \\
\hline Fluthiacet + AMS + NIS & 0.06 & $17+0.01$ & 520 & 5,287 & $9,099 \mathrm{abc}$ & $13,971 \mathrm{~b}-\mathrm{f}$ & 28,678 & 11,337 & 4,108 & 3,372 & $76,698 \mathrm{ab}$ \\
\hline Cloransulam-methyl + AMS + NIS & 0.4 & $22+0.0025$ & 557 & 3,678 & $7,807 \mathrm{bcd}$ & 16,575 a-e & 28,186 & 13,622 & 4,990 & 5,449 & $81,410 \mathrm{ab}$ \\
\hline Flumiclorac + COC & 0.6 & 16 & 643 & 4,811 & 7,580 b-e & $14,792 \mathrm{a}-\mathrm{f}$ & 28,976 & 11,451 & 5,390 & 7,467 & $81,643 \mathrm{ab}$ \\
\hline Thifensulfuron-methyl + AMS + NIS & 0.04 & $22+0.0025$ & 358 & 5,466 & 7,466 b-e & $19,543 \mathrm{a}$ & 32,495 & 9,230 & 3,204 & 6,286 & $84,360 \mathrm{ab}$ \\
\hline Tribenuron-methyl + AMS + NIS & 0.2 & $22+0.0025$ & 755 & 4,368 & 7,403 b-e & 16,299 a-e & 30,252 & 8,711 & 5,487 & 6,169 & $79,633 \mathrm{ab}$ \\
\hline Amin & 0.9 & 0.0025 & 521 & 6,231 & 5,260 e & $10,457 \mathrm{f}$ & 21,574 & 7,276 & 5,308 & 3,600 & $60,758 \mathrm{c}$ \\
\hline Metsulfuron-methyl + NIS & 0.1 & 0.0025 & 490 & 6,770 & 7,395 b-e & 12,814 ef & 25,772 & 12,086 & 5,938 & 5,842 & $78,216 a b$ \\
\hline
\end{tabular}

${ }^{a}$ Means within columns followed by the same letter are not significantly different according to Fisher's protected LSD test at $P \leq 0.05$. No significant differences within a column were observed when no letters are included.

${ }^{\mathrm{b}} \mathrm{B}$ size potatoes include those with a diameter of $4.4 \mathrm{~cm}$ or less.

${ }^{c}$ AMS rates are in $\mathrm{g} \mathrm{ha}^{-1}$. COC, MSO, and NIS rates are in \% v/v.

${ }^{\mathrm{d}}$ Abbreviations: AMS, ammonium sulfate; COC, crop oil concentrate; MSO, methylated seed oil; NIS, non-ionic surfactant. 
potato plants treated with cloransulam-methyl, thifensulfuron methyl, tribenuron-methyl, or metsulfuron-methyl. Where tribenuron-methyl was applied, the cull yield was almost as high as the Wisconsin state average total marketable yield for the 2014 production year (about $48,000 \mathrm{~kg} \mathrm{ha}^{-1}$ ) (USDA-NASS 2015). The yield of tubers in the largest grade categories was lowest where tribenuronmethyl and metsulfuron-methyl were applied, with no tubers weighing more than $454 \mathrm{~g}$ and reduced yield in the 283 to $368 \mathrm{~g}$ weight category compared to the yield of the non-treated potato plants. Total tuber yield from the plants treated with these two herbicides was also reduced compared to the yield of the non-treated potato plants (Table 5).

Daughter Potato Plant Growth. Tubers from the mother plants that were exposed to the herbicide treatments were planted as seed in the following season, and the daughter plants were monitored for evidence of herbicide injury and harvested to evaluate tuber yield and quality. In 2014, for the daughter plants grown from the 2013 mother plants, stand density and the number of plants expressing herbicide symptoms in all herbicide treatment groups was similar to that of the non-treated group. Visual estimations of potato foliar injury were $10 \%$ or $11 \% 42 \mathrm{~d}$ after planting (DAP) where dicamba, glyphosate at the lowest application rate, fluthiacet, or aminopyralid were applied to the mother plants in 2013. All other visual observations of potato foliar injury were lower than $10 \%$, and injury was minimal in all herbicide treatments by 48 DAP (Table 6). The severity of herbicide injury and negative effect on tuber yield and quality observed in the mother plant study in 2014 persisted into the 2015 daughter plant study for several treatment groups. Stand density at 35 and 43 DAP was reduced where plants were treated with aminopyralid compared to the non-treated plots. The number of potato plants expressing herbicide symptomology 49 DAP was greater than $50 \%$ of the planted crop density in glyphosate (lowest rate) or aminopyralid treatment plots. Visual estimation of crop injury 43 DAP was $10 \%$ or greater where glyphosate was applied at the two highest rates and where mesotrione or metsulfuron-methyl was applied, and $50 \%$ or greater where dicamba or aminopyralid was applied. Injury from dicamba, glyphosate at the highest rate, and aminopyralid persisted 49 DAP and ranged from $30 \%$ to $54 \%$ (Table 7 ).
Daughter Potato Plant Yield. Potato seed planted in 2014 from the 2013 mother plant study exhibited minimal injury, and stand density was similar for the treated and non-treated potato plants. As a result, tuber yield and quality in the 2014 harvest was similar among all herbicide treatments and between the treatments and the non-treated check (Table 8). In 2015, however, some of the herbicides that had caused injury in the 2014 mother plants also reduced yield in the 2015 daughter crop. Tuber quality, indicated by the cull weight, did not differ among treatments or between treatments and the nontreated check. Glyphosate applied at the lowest rate increased tuber yield in the 57 to $112 \mathrm{~g}$ weight category. Injury from aminopyralid reduced the yield of the two lightest grade weight categories, and as a result reduced the total tuber yield compared to the non-treated check. Additionally, metsulfuron-methyl reduced tuber yield in the 113 to $169 \mathrm{~g}$ weight category compared the non-treated check. All other weight categories and total tuber yield were similar between herbicide-treated potato plants and the non-treated plants (Table 9).

The variability among production years in potato response and daughter tuber injury documented by Wall (1994) for the herbicides clopyralid, tribenuron, and dicamba was similar to what was observed in this study with a broader array of herbicide active ingredients. The lack of consistency in the connection between visual injury in the mother potato plant and affected daughter tuber growth and yield in the following year challenges traditional crop scouting as a tool to assess off-target herbicide risk. While somewhat variable, these results document that seed potato crops are at risk if off-target herbicide exposure does occur. Our current research is focused on non-visual measurement of potato injury, such as through spectral image sensing, that could be used in greenhouse assays during the time period between the mother plant seed production year and the following daughter tuber field season to predict risk of herbicide-induced growth issues.

\section{Literature Cited}

Arledge-Keene A, Mitchell P (2010) Economic Impact of Specialty Crop Production and Processing in Wisconsin, UW-Madison Agricultural and Applied Economics Working Paper. https://www.aae.wisc.edu/pubs/misc/docs/mitchell.crop. impacts.pdf. Accessed February 24, 2016 
Colquhoun J, Gevens A, Groves R, Heider D, Jensen B, Nice G, Ruark M (2016) Commercial Vegetable Production in Wisconsin, University of Wisconsin Extension Bulletin A3422. http://learningstore.uwex.edu/assets/pdfs/A3422.PDF. Accessed February 24, 2016

Dean B, Thornton R (1992) The Specific Gravity of Potatoes. Pullman, WA: Washington State University, Cooperative Extension Bulletin 1541. 20 p

Eberlein C, Westra P, Haderlie L, Whitmore J, Guttieri M (1997) Herbicide Drift and Carryover Injury in Potatoes. Moscow, ID: Pacific Northwest Extension Publication 498. 15 p

Felix J, Boydston R, Burke I (2011) Potato response to simulated glyphosate drift. Weed Technol 25:637-644

Hutchinson J, Felix J, Boydston R (2014) Glyphosate carryover in seed potato: effects on mother crop and daughter tubers. Am J Potato Res 91:394-403

Olszyk D, Pfleeger T, Lee E, Plocher M (2010) Potato (Solanum tuberosum) greenhouse tuber production as an assay for asexual reproduction effects from herbicides. Environ Tox Chem 29:111-121
[USDA-AMS] US Department of Agriculture, Agricultural Marketing Service (2013) United States Standards for Grades of Potatoes for Processing. http:// www.ams.usda.gov. Accessed October 15, 2013

[USDA-ERS] US Department of Agriculture, Economic Research Service (2016) Potatoes. http://www.ers.usda.gov/topics/crops/ vegetables-pulses/potatoes.aspx. Accessed February 24, 2016

[USDA-NASS] US Department of Agriculture, National Agricultural Statistics Service (2015) Potatoes 2014 Summary. http://usda.mannlib.cornell.edu/usda/current/Pota/Pota-09-172015.pdf. Accessed February 26, 2016

Wall D (1994) Potato (Solanum tuberosum) response to simulated drift of dicamba, clopyralid, and tribenuron. Weed Sci 42:110-114

Worthington T (1985) The effect of glyphosate on viability of seed potato tubers. Potato Res 28:109-112

Received May 10, 2016, and approved September 22, 2016.

Associate Editor for this paper: Steve Fennimore, University of California, Davis. 